\title{
Knowledge of HIV infected individuals about prevention of cryptosporidiosis
}

\author{
P M Slade, J S Kepple, D R Churchill
}

Cryptosporidiosis is the commonest cause of chronic diarrhoea in extensively investigated patients with advanced HIV infection in the United Kingdom. ${ }^{1}$ There is currently no effective treatment. The principal risk for acquiring infection with cryptosporidiosis is from drinking contaminated water. The infection may also potentially be acquired in a number of other ways, including through sexual exposure (particularly oro-anal contact); from exposure to infected animals; swimming in contaminated water; and eating unwashed fruit and vegetables. In 1995 guidelines on prevention of cryptosporidiosis in immunocompromised patients were issued in the United Kingdom by the chief medical officer ${ }^{2}$ and in the United States by the Centers for Disease Control and Prevention (CDC). ${ }^{4}$ The guidelines advised that HIV infected patients with CD4+ lymphocyte counts below 200 cells $\times 10^{6} / 1$ should boil all drinking water for at least 1 minute. They also suggested that patients with $\mathrm{CD} 4+$ lymphocyte counts above 200 cells $\times 10^{6} / 1$ should consider boiling water for drinking, although they are at lower risk of chronic diarrhoea. Alternatively, the use of submicron filters in water supplies can give protection against cryptosporidiosis, provided the filters are of suitable specification and serviced appropriately. ${ }^{4}$ Use of bottled water may reduce the risk of infection, but methods of disinfection of bottled water vary, and some brands may contain oocysts of Cryptosporidium. We used a semistructured questionnaire to assess the degree to which HIV infected individuals attending St Mary's Hospital were aware of, and followed, these guidelines.

One hundred questionnaires were issued to unselected patients in November 1996; there had been no prior systematic campaign to educate patients about prevention of cryptosporidiosis. Fifty nine questionnaires were returned. Respondents were mostly male (96\%) with a mean age of 38 (range 24-60) years, and mean CD4+ count of $308 \times 10^{6 / 1}$ (range $\left.0-1900 \times 10^{6} / 1\right)$. Of the 45 respondents who knew their latest CD4+ count, $17(38 \%)$ had counts of $200 \times 10^{6} / 1$ or below. Nine patients had never heard of cryptosporidiosis and offered no responses. Two of 50 (4\%) participants had had a diagnosis of cryptosporidiosis previously. Transmission of infection was poorly understood. Only 23/50 (46\%) realised that tap water posed an infection risk. Twenty three of $50(46 \%)$ felt it was sufficient to boil their drinking water for 30 seconds or less to eliminate Cryptosporidium (compared with the CDC guidelines of at least 1 minute). Twenty eight of $50(56 \%)$ correctly identified sexual contact as a potential risk, but $30 / 50(60 \%)$ also believed that intravenous drug use constituted a risk of infection. Few patients took adequate steps to prevent infection. Only $6 / 50$ $(12 \%)$ reported always boiling their drinking water, with a further $12 / 50(24 \%)$ boiling their water only occasionally.

Only $11 / 59$ (19\%) of respondents reported having received any information about the condition, with $6 / 59$ (10\%) claiming to have been given information from their doctor, $3 / 59$ (5\%) from information newsletters, $2 / 59$ (3\%) from nurses involved in their care, and $2 / 59$ (3\%) from their dietitian. However, respondents who reported having received information about the condition scored no better than those who had not.

Thirty four of the 95 (36\%) questionnaires issued to staff involved in the care of HIV patients were returned. Transmission of infection was better understood by staff, with all respondents being aware that tap water posed a threat of infection. Only 14/34 (41\%) were aware that sexual contact was a potential risk. Measures to prevent infection were also better uriderstood among staff, although $8 / 34$ (24\%) still felt that it was sufficient to boil drinking water for a shorter period than that recommended in the CDC/CMO guidelines. The individual "scores" achieved by staff (mean $70 \%$ correct responses) were consistently higher than those achieved by the patients (mean 50\%). We conclude that the education of patients at risk of cryptosporidiosis is inadequate, and few patients thus take steps to avoid infection. Unlike many prophylactic measures accepted and adopted for other opportunistic infections, avoidance of cryptosporidiosis is a cheap and safe measure. More widespread and intensive education of patients and staff is needed to ensure the risk of cryptosporidiosis in immunocompromised patients is minimised.

1 Blanshard C, Francis N, Gazzard BG. Investigation of chronic diarrhoea in acquired immunodeficiency syndrome. A prospective study of 155 patients. Gut 1996; 39:824-32.

2 Department of the Environment, Department of Health Cryptosporidium in water supplies. Second report of the group of experts. London: HMSO, 1995.

3 Chief Medical Officer. Advice to boil water to prevent cryptosporidiosis in immunocompromised patients. CMO's Update 1995:8.

4 Centers for Disease Control and Prevention USPHS/IDSA guidelines for the prevention of opportunistic infections in persons infected with opporimmunodeficiency virus: $1-34$.

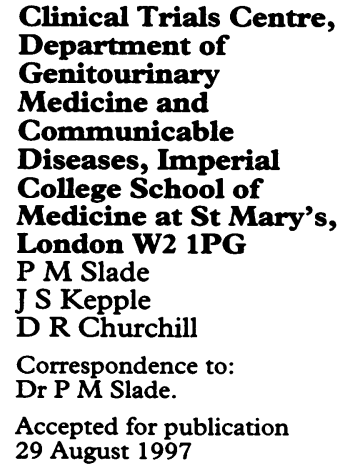

